\title{
Gene Therapy for Neurodegenerative Diseases
}

\author{
Vivek Sudhakar $^{1} \cdot$ R. Mark Richardson ${ }^{1}$ (if \\ Published online: 12 December 2018 \\ (C) The American Society for Experimental NeuroTherapeutics, Inc. 2018
}

\begin{abstract}
Gene therapy has the potential to provide therapeutic benefit to millions of people with neurodegenerative diseases through several means, including direct correction of pathogenic mechanisms, neuroprotection, neurorestoration, and symptom control. Therapeutic efficacy is therefore dependent on knowledge of the disease pathogenesis and the required temporal and spatial specificity of gene expression. An additional critical challenge is achieving the most complete transduction of the target structure while avoiding leakage into neighboring regions or perivascular spaces. The gene therapy field has recently entered a new technological era, in which interventional MRI-guided convection-enhanced delivery (iMRI-CED) is the gold standard for verifying accurate vector delivery in real time. The availability of this advanced neurosurgical technique may accelerate the translation of the promising preclinical therapeutics under development for neurodegenerative disorders, including Parkinson's, Huntington's, and Alzheimer's diseases.
\end{abstract}

Key Words Gene therapy · Parkinson's disease $\cdot$ Huntington's disease $\cdot$ Alzheimer's disease $\cdot$ viral vector $\cdot$ intraoperative MRI.

\section{Introduction}

Neurodegenerative diseases affect a large portion of our increasingly elderly population worldwide. Idiopathic Parkinson's disease (PD) impacts over one million people in the USA alone, with $1 \%$ of individuals older than 60 likely to develop PD, whereas an estimated 5.7 million Americans of all ages are living with Alzheimer's dementia. Current therapies for neurodegenerative disorders, however, are aimed at symptomatic relief and do not address the underlying pathology. As neurodegeneration progresses, the efficacy of pharmacological treatments is reduced. Because the blood-brain barrier prevents significant amounts of most systemically administered agents from reaching therapeutic parenchymal levels without producing toxicity, significant side effects typically increase with escalating medication dosage. Direct intracerebral delivery of therapeutics is a strategy that circumvents the blood-brain barrier to target the disease process with functional-anatomic specificity. Therapeutic molecules, such

R. Mark Richardson richardsonrm@upmc.edu

1 Brain Modulation Laboratory, Department of Neurological Surgery, University of Pittsburgh School of Medicine, Pittsburgh, Pennsylvania 15213, USA as growth factors, can be delivered directly into the brain parenchyma, or alternatively, the genes that code for these types of molecules can be delivered. The former approach requires chronic re-administration of the therapeutic agent, whereas a single-gene therapy infusion may have durability on the order of a decade or more.

Neurodegenerative disease processes often involve the progressive accumulation of dysfunctional proteins within cells, leading to cell death. There are no existing therapies that correct underlying neurodegenerative disease processes; current therapies provide merely symptomatic relief. As the underlying disease process progresses, the efficacy of pharmacological treatments is reduced, despite increases in medication dosage. Thus, the side effect to benefit ratio increases. Intracerebral drug delivery, specifically gene therapy, is a promising strategy for mitigating these deficiencies in medical management. By altering or inducing the expression of specific proteins, gene therapy may allow for neuroprotection, neurorestoration, and ultimately, correction of the underlying pathogenic mechanism.

Appropriate gene therapy parameters, however, depend on disease pathogenesis and the temporal evolution of the pathological phenotype. In addition, the temporal and spatial specificity of gene expression must be considered. Temporal specificity in gene therapy refers to whether gene expression is constitutive or regulated, whereas spatial specificity refers to 
whether and how the gene expression is confined to a particular brain region or cell type. Focal neurosurgical delivery of gene therapy vectors circumvents the blood-brain barrier and allows for therapy to be specified to anatomical regions of interest, avoiding exposure to other brain areas in which transgene expression is not necessary or is undesired [1]. Although gene expression under control of specific promoters potentially can be regulated by medication (rather than "always on") and delivered to specific cell groups (allowing for neuronal phenotype-specific effects), the current state of the art in human gene therapy trials does not involve regulated or phenotype-specific gene expression.

\section{Delivery of Gene Therapy}

\section{Viral Vectors}

Modified viral vectors are used to delivery genes of interest to the brain, with vectors derived from adeno-associated viruses (AAVs) having been used almost exclusively in clinical trials for CNS disorders. One exception is lentiviral vectors, which can carry a larger DNA payload, and have been used in one clinical trial to date [2]. AAVs can be subdivided into serotypes, based on their capsid profiles. Viral vector particles are transported within the brain from one region to another, either in an anterograde manner (transduction of cells in a brain region that receives projections from the infusion site), retrograde manner (transduction of cells in a brain region that sends projections to the injection site), or both. The manner of this transport depends on the serotype, and in the case of preclinical experiments, on the transduced species [3]. Understanding how a specific serotype distributes gene products from the targeted region for vector delivery is crucial for creating a predictable and reproducible gene therapy paradigm [4-9]. Due to its relative phenotype specificity for neurons and clinical safety profile, AAV2 has been the gold standard for neurosurgical gene therapy and has been used in multiple phase 1 [10-14] and phase 2 studies [15, 16] (Tables 1 and 2). Given that AAV2 has a robust preference for anterograde transportation of the gene product [9], when using AAV2 vectors, one must assume that downstream targets of projection neurons also will express the transgene.

\section{Surgical Platform}

A gold standard platform for neurosurgical delivery of gene therapy vectors now exists, following more than two decades of preclinical and clinical work. The development of convection-enhanced delivery (CED) in the 1990s allowed for efficient, direct, and controlled distributions of viral vector particles throughout the brain. First described by Oldfield et al. [17], CED utilizes a pressure gradient to generate the 


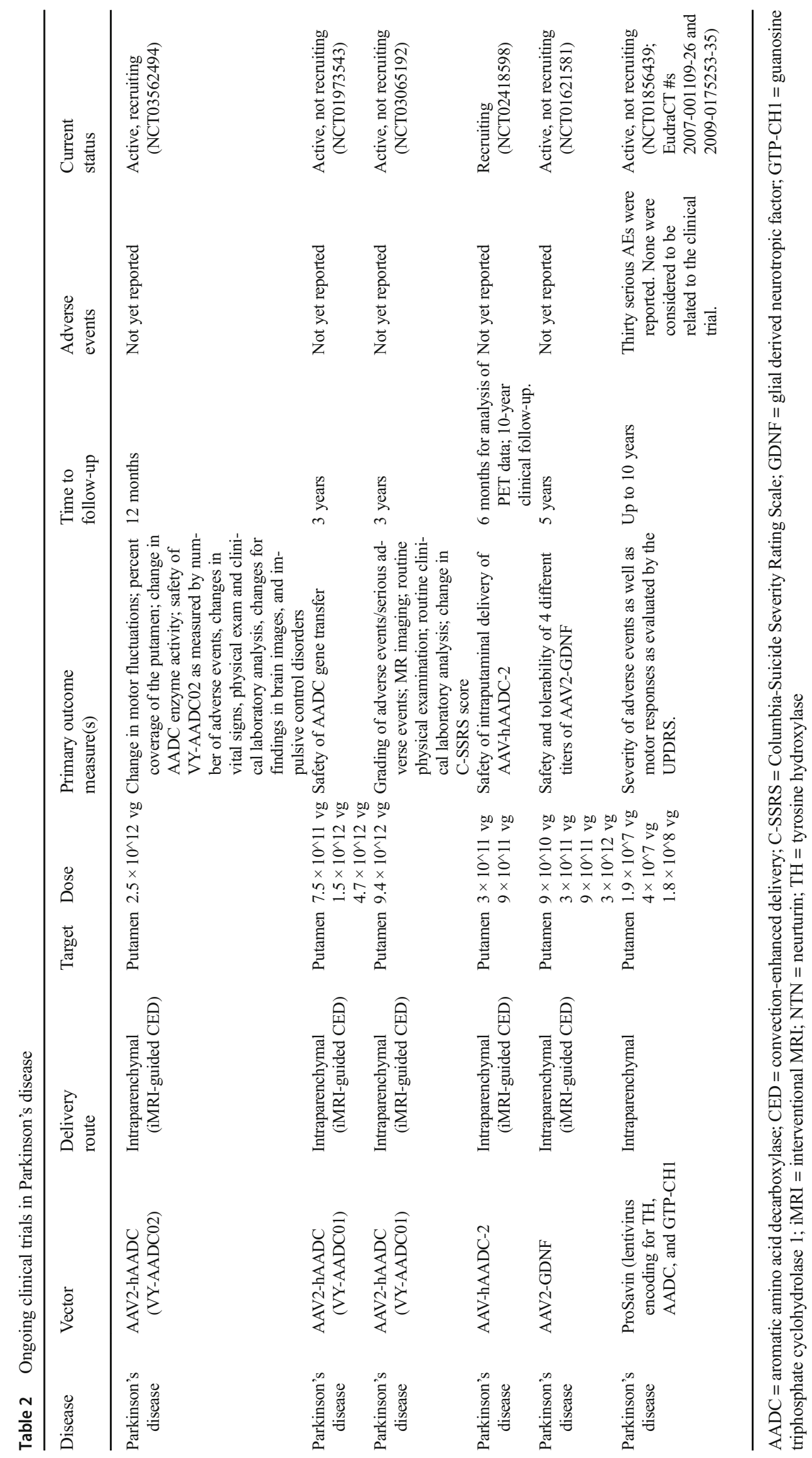


Fig. 1 MRI-compatible infusion cannula for clinical CED. A twostep design cannula with a ceramic body and inner silica sleeve designed to allow low priming volumes. The ceramic body is encased with a protective outer polymer sleeve (MRI Interventions, Inc.)

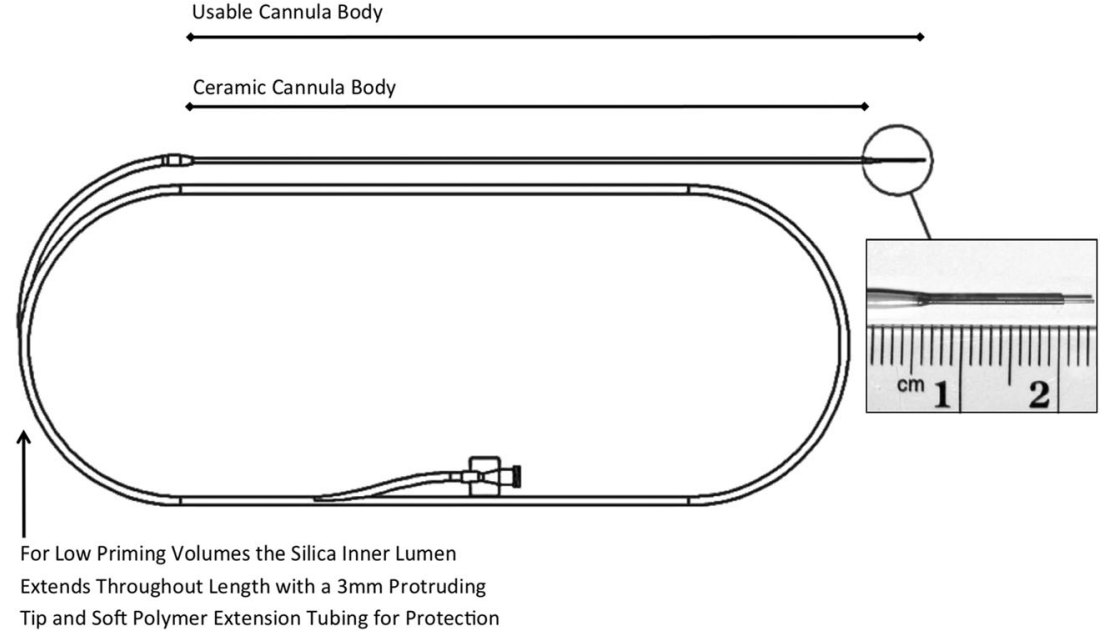

bulk flow of infusate within the interstitial fluid space. Because CED relies on fluid convection rather than passive diffusion to achieve distribution, it can uniformly deliver high concentrations of macromolecules over significant volumes. Subsequent development of an infusion cannula with a step close to the cannula tip allowed positive pressure to be exerted without significant reflux [18]. Stereotactic infusions nonetheless occurred in a black box, as there was no method to verify whether the infusate was distributed within the target or leaked into neighboring regions or back up the cannula track.

Similarly, stereotactic infusions employed in clinical trials previously have occurred without a method for verifying the extent to which the infusate is distributed within the target during the course of the infusion, versus leaking into neighboring regions or refluxing up the cannula track. For this reason, the failure of protein infusion and gene therapy trials in PD is thought to be due in large part to inadequate delivery. The development of real-time MRI-guided CED using interventional or intraoperative MRI (iMRI-CED) seeks to overcome these challenges by enabling monitoring of the infusion via co-administration of an MRI contrast agent mixed with the therapeutic drug. Studies in nonhuman primates have allowed for quantification of CED dynamics and the intraoperative detection of leakage and reflux [19-22], definition of structure-specific "green zones" delineated as reliable cannula placement zones [23, 24], and verification that gadoteridol distributions visible on MRI correspond well with subsequent transgene expression patterns $[25,26]$. These results set the stage for planning cannula placement with respect to individual patients' anatomy, and led to the development of a clinical platform for iMRI-CED [25, 27].

Currently, the sole commercially available MRIcompatible drug delivery platform (MRI Interventions, Irvine, CA) includes a skull-mounted aiming device (SmartFrame®), a reflux-resistant CED cannula (SmartFlow®) (Fig. 1), and an MRI-integrated software package (ClearPoint $\AA)$ that communicates with both the console and the operating neurosurgeon in the MRI suite. The key features of this platform are 1) patient positioning on the MRI gantry under general anesthesia; 2) definition of target coordinates with respect to the MRI isocenter rather than to a separate stereotactic space; 3 ) trajectory alignment and cannula insertion via a burr hole-mounted trajectory guide in place of a traditional stereotactic frame-based system; 4) integration of planning, insertion, and real-time MRI confirmation of cannula placement and subsequent infusate distribution during a single procedure; 5) cannula design involving a small inner lumen to allow for low priming volume and minimal waste of therapeutic agents, and a two-step tip that minimizes drug reflux. The accuracy of the ClearPoint system was first validated preclinically in nonhuman primate infusion studies [25], and subsequently has been validated extensively for the clinical placement of deep brain stimulation leads, with average targeting errors on the order of $1 \mathrm{~mm}$ [28-31]. This platform enables cannula placement to be aligned precisely with the planned trajectory, optimizing the ability to use the two-step design for controlling the start of the infusion within the target structure (Fig. 2).

\section{Preclinical Studies to Clinical Trials}

\section{Parkinson's Disease}

Two treatment strategies have been employed thus far in AAV2-mediated gene therapy clinical trials for PD. First, symptomatic therapies aimed at improving clinical symptoms rather than altering the course of the disease. Two strategies have been employed, which involved gene transfer of either 1) glutamic acid decarboxylase (GAD), which catalyzes the synthesis of $\gamma$-aminobutyric acid (GABA) from glutamate or 2) aromatic L-amino acid decarboxylase (AADC), a rate-limiting enzyme for dopamine synthesis that converts levodopa to dopamine. Second, neurorestorative therapies, focused on 


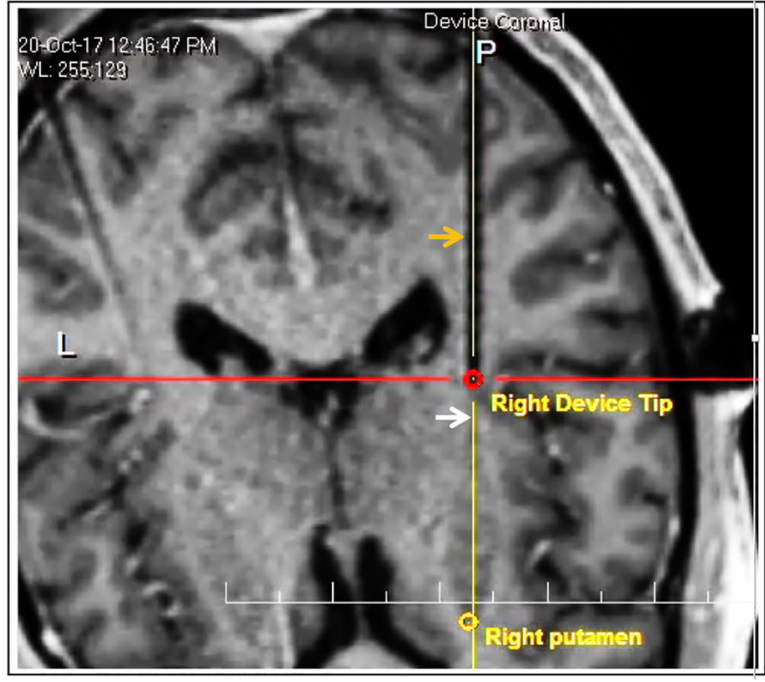

Fig. 2 Initial position of infusion cannula. Orange arrows indicate the artifact from the infusion cannula and white arrows indicate the border of the putamen, in axial (left) and sagittal (right) views. The red circle indicates the location of the second step, which is $\sim 8 \mathrm{~mm}$ from the putamen border, placing the tip $\sim 5 \mathrm{~mm}$ into the target structure. Initial

attempting to restore cellular functions affected by the disease process, have involved in vivo gene transfer of a neurotrophic factor, either 1) glial derived neurotropic factor (GDNF) or 2) neurturin.

\section{AAV2-GAD}

The AAV2-GAD strategy was focused on delivering GAD to the subthalamic nucleus (STN) in an effort to increase local GABA inhibition and correct pathological overactivity in this basal ganglia node. Although injecting a GABA receptor agonist, muscimol, into the STN had been observed to reduce PD motor symptoms [32], when the delivery of AAV2-GAD to the STN of MPTP-lesioned nonhuman primates was eventually tested, no improvement in clinical rating scales was seen [33]. Nonetheless, phase I and phase II trials were performed in which AAV2-GAD was delivered, via CED, to the STN of PD patients [16, 34]. Phase I trials involved 12 patients who received a unilateral infusion of $50 \mu \mathrm{L}$ AAV2GAD into the STN of the more symptomatic hemisphere, in three different dosing groups: $1 \times 10^{11} \mathrm{vg} / \mathrm{mL}, 3 \times 10^{11} \mathrm{vg} /$ $\mathrm{mL}$, and $1 \times 10^{12} \mathrm{vg} / \mathrm{mL}$. Subjects demonstrated improvements in their Unified Parkinson's Disease Rating Scale (UPDRS) scores up to 12 months post-infusion. FDG-PET scans confirmed the efficacy of the gene transfer, as there was a decrease in activity of the STN [34]. In the phase II trial, 16 subjects received bilateral infusions of AAV2-GAD $(1 \times$ $10^{12} \mathrm{vg} / \mathrm{mL}$ ) and were assessed after 6 months. Patients displayed improvements in their PD motor symptoms, but not to any greater extent than could be achieved following deep brain stimulation of the STN [16].

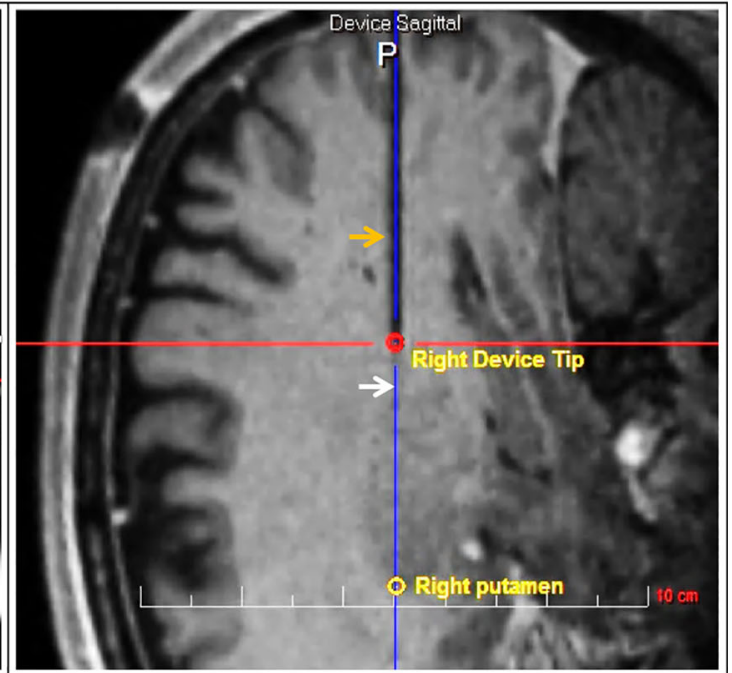

reflux up to the first step will confine the initial infusion volume within the putamen. Further insertion of the cannula such that the second step reaches the border of the putamen will allow for further spherical expansion of the infusion volume within the target

\section{AAV2-AADC}

In PD patients, dopamine replacement therapy is efficacious early in the disease for controlling symptoms. As the disease progresses, however, increasing doses of levodopa fail to provide the same therapeutic benefit, often resulting in severe motor fluctuations and levodopa-induced dyskinesias. AADC gene therapy is a pro-drug approach, in which the anatomically focal replacement of the hAADC enzyme enables a high level of conversion of levodopa to dopamine, in the setting of continued use of levodopa medication. The postcommissural (sensorimotor territory) striatum has been the chosen target for this therapy as medium spiny neurons within the striatum do not deteriorate in PD, and studies have demonstrated the ability of these cells to express transgenes for long periods of time $[35,36]$. Preclinical studies demonstrated that hAADC delivery to MPTP-lesioned primates produced statistically significant long-term improvements in behavioral rating scores, significantly lowered levodopa requirements, and reduced levodopa side effects [35-39]. In addition, $\left[{ }^{18} \mathrm{~F}\right]$-fluoro-L- $m$-tyrosine (FMT; a marker of AADC activity) PET imaging of Parkinsonian nonhuman primates (NHPs) who received hAADC demonstrated continued overexpression of AADC at 9 years post-infusion, indicating the longterm efficacy of this therapy $[35,36]$.

A phase 1 clinical trial explored the delivery of AAV2hAADC in 10 patients who subsequently were monitored for up to 5 years $[10,12,40]$. One hundred microliters of vector was delivered per hemisphere in two sites in each putamen, with two dose cohorts $\left(9 \times 10^{10} \mathrm{vg}\right.$ and $\left.3 \times 10^{11} \mathrm{vg}\right)$. Six-month postoperative FMT-PET scans demonstrated a 
$25 \%$ and $65 \%$ increase in the uptake levels of AADC in the low- and high-dose groups respectively [40]. This study demonstrated the safety of this therapeutic strategy, with modest efficacy, but was not continued into further phases by the industry sponsor. Analysis of immediate postoperative T2 images demonstrated that coverage of the target region likely was quite variable [22]. Nonetheless, the elevated PET signal observed in the first 12 months persisted over 4 years in both dose groups [40]. The off-medication UPDRS, however, displayed a slow deterioration after the first year, suggesting the need for a controlled efficacy trial with higher vector doses, using real-time MRI visualization of vector distribution.

A new phase I trial is currently underway to deliver AAV2hAADC to the putamen, this time employing iMRI-CED (ClinicalTrials.gov: NCT01973543). Infusion volumes have been significantly increased, and up to $8.8 \times 10^{12}$ vector genomes will be delivered per subject. Enrollment in phase $1 \mathrm{~b}$ studies was recently completed at the University of California, San Francisco and the University of Pittsburgh Medical Center. The use of iMRI-CED results in a remarkable ability to track and dynamically modulate vector infusions in real time (Fig. 3) and allows for precise calculations of the volume of vector coverage infused into the target brain region. Phase 2 studies are ongoing.

The results of a two-center (Créteil, France, and Cambridge, UK) phase $1 / 2$ open-label trial of ProSavin, a lentiviral vector that encodes not only AADC, but the other rate-limiting dopamine biosynthetic enzymes tyrosine hydroxylase and cyclohydrolase 1, were reported in 2014 [2]. Although lentiviral vectors have the advantage of being able to carry a larger genetic payload than AAV vectors, no clinical trial using in vivo administration of lentiviral vectors in any human disease had previously been reported. In this open-label, dose-escalation study with 12-month follow-up, 3 doses of ProSavin were assessed in 4 patient cohorts with bilateral delivery to the putamen. Increased on-medication dyskinesia was the most common adverse event, which resolved in each case with reduction of dopaminergic medication, but off-medication dyskinesias were not observed. Offmedication UPDRS motor scores were significantly reduced at both 6 and 12 months in all patients, relative to their baselines (33\% and $31 \%$, respectively); however, no significant difference was seen between the different dose cohorts.

The therapeutic goal of this gene therapy strategy was to produce a continuous and stable production of dopamine in the motor region of the putamen. Evaluation of this effect by positron emission tomography, however, produced conflicting results. There was no effect in ${ }^{18} \mathrm{~F}$-levodopa uptake, an analog of levodopa used to evaluate striatal dopaminergic presynaptic function, after ProSavin administration. In contrast, [11C]raclopride binding, which assesses the degree of dopamine binding to the $\mathrm{D} 2$ dopamine receptor, did increase in a dose-dependent manner. Similarly, there was no dosedependent effect on UPDRS motor scores, despite the fact that the authors switched to a continuous infusion method, increased the delivery rate to $3 \mu \mathrm{L} / \mathrm{min}$, and decreased the
Fig. 3 Real-time visualization of simultaneous bilateral MRIguided infusion of AAV2-AADC (1:1 admixture with gadoteridol) to the putamen (blue shading in panel a). (a) Infusions are expected to grow concentrically after reaching the first step. (b) The cannula is advanced in $\sim 2$ $3 \mathrm{~mm}$ increments, with a goal of filling the structure from proximal to distal. (c) A second step acts as an additional temporary barrier to infusion volume growth along the cannula axis. (d) An axial view demonstrates appropriate coverage of the putamen, on each side, at the end of the drug delivery procedure

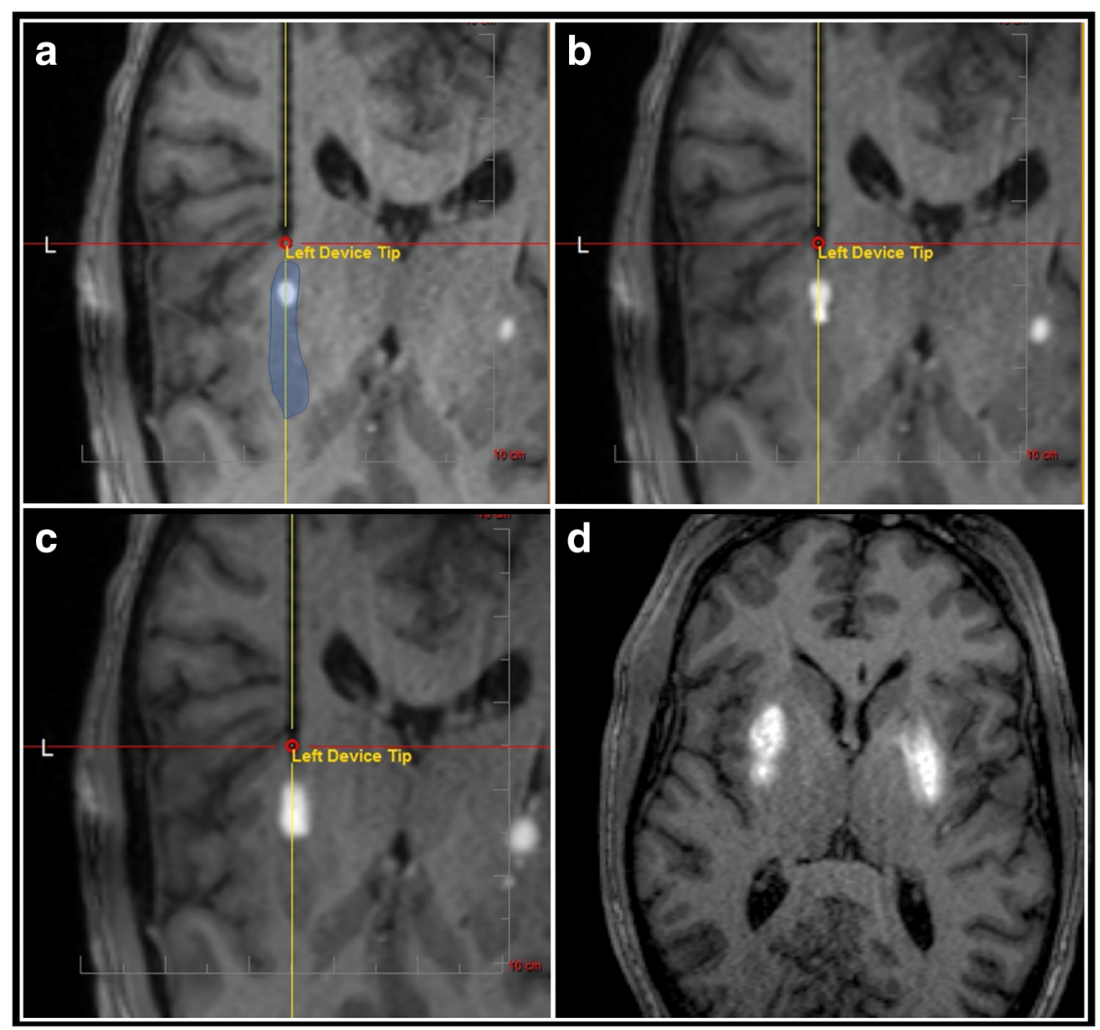


cannula diameter in between the second and third cohorts to increase the rate of delivery and enhance the distribution of the vector. The authors' conclusion was that they could not proceed to a more definitive double-blind placebo-controlled trial until possessing an optimum mode and dose of delivery, essentially acknowledging that without real-time MRI during infusion of the viral vector, it is not possible to determine accurate and adequate delivery. Nonetheless, this work demonstrated the safety of this first-in-man use of a lentiviralbased vector for a central nervous system disorder.

\section{GDNF}

Neurorestorative therapies are aimed at modifying underlying disease processes. Multiple restorative neurotrophins have been identified, and of them, GDNF has shown the most promise in ameliorating Parkinson's pathology in animal models [41]. GDNF and related neurotrophins are members of the transforming growth factor- $\beta$ (TGF- $\beta$ ) superfamily. Two GDNF delivery paradigms have been developed and evaluated in preclinical and clinical trials: direct protein infusions and AAV-mediated gene transfer. In clinical trials, intrastriatal infusions of GDNF protein [42], despite positive open-label phase I findings [43, 44], failed to meet primary endpoints in double-blind phase II testing, and were halted after the development of hardware complications and the detection of neutralizing antibodies against recombinant GDNF in the cerebrospinal fluid. Analysis of this trial revealed that majority of GDNF failed to migrate far from the catheter tip, leading to minimal coverage of the putamen [45]. A new study of intermittent glial GDNF infusions administered via CED currently is underway in the UK (EudraCT\# 2011-00386634). This trial tests a recently developed platform consisting of a transcutaneous bone-anchored port and implantable multi-catheter system for chronic intermittent CED [46].

The first reported preclinical GDNF gene therapy experiments employed a lentiviral vector, delivered to the striatum and substantia nigra of aged normal and MPTP-lesioned monkeys [47]. These studies provided evidence for the prevention of further nigrostriatal degeneration and for the sprouting of degenerated fibers, and animals demonstrated no immunological response and continued GDNF expression 8 months following infusion. Subsequently, multiple studies have investigated the safety and efficacy of delivering larger doses of AAV2-GDNF to the normal and MPTP-lesioned NHP brain [48-50]. Importantly, Bankiewicz and colleagues altered the experimental model to more accurately match the clinical presentation of PD [50]. They delivered the viral vector only after PD symptoms had been present for more than 6 months, rather than delivering the gene within weeks of creating the MPTP lesion, a timeframe in which GDNF could still act in a protective rather than restorative fashion. In monkeys having PD symptoms for 6 months, subsequent putamenal AAV2-
GDNF delivery resulted in significantly improved clinical rating scores, increased PET-FMT uptake, and increased DA turnover. Building from this work, combined with advances by Bankiewicz and colleagues using interventional MRIguided CED [27], an NIH-funded phase 1 trial was initiated at the National Institutes of Health Clinical Center to deliver four different doses of AAV2-GDNF to patients with advanced Parkinson's disease (ClinicalTrials.gov: NCT01621581).

\section{Neurturin}

Neurturin (NTN) is a functional and structural homolog of GDNF that has been reported to bind to both the GDNF receptor $(\mathrm{GFR} \alpha 2)$ and the GFR $\alpha 1$ receptor [51]. The potential for neurturin to act as a neuroprotective agent, similar to that of GDNF, was tested in NHPs who received an infusion of AAV2-NTN 4 days following MPTP infusion in the striatum and substantia nigra [52]. A progressive recovery of function occurred, starting 4 months post-infusion and persisting until the end of the study (10 months), in conjunction with histological evidence of preserved nigrostriatal dopaminergic innervation. AAV2-NTN delivery to the human putamen subsequently was explored in phase I and phase II clinical trials [11, $15,53]$. Twelve phase I patients received bilateral infusions of AAV2-NTN (labeled CERE-120 by the industry sponsor) to the putamen, in two dosing groups: low dose $\left(1.4 \times 10^{11} \mathrm{vg} /\right.$ $\mathrm{mL})$ and high dose $\left(5.7 \times 10^{11} \mathrm{vg} / \mathrm{mL}\right)$, each of which received only $40 \mu \mathrm{L}$ of vector, delivered through a Hamilton syringe without CED. There were no serious adverse effects, and patients displayed a significant UPDRS motor score improvement 6 months following the infusion (27.9\% reduction in the low-dose group and $51.2 \%$ in the high-dose group).

Based on these results, a 12-month double-blind sham surgery-controlled phase II trial was conducted with the same methodology, and 34 patients were enrolled who received $40 \mu \mathrm{L}$ per hemisphere of AAV2-NTN $\left(5.7 \times 10^{11} \mathrm{vg} / \mathrm{mL}\right)$. This cohort, however, did not experience clinically significant improvements, compared to a placebo group, at 12 months following the infusion [15]. The study investigators hypothesized that the lack of clinical efficacy resulted from failure of retrograde transport of the vector from the putamen to the substantia nigra and conducted a second phase I study with AAV2-NTN, in which the vector was delivered to the substantia nigra of PD patients [54]. Following an adequate demonstration of safety, a phase II trial was then performed, targeting both the substantia nigra and the putamen $(2.0 \times$ $11 \mathrm{vg}$ and $1.0 \times 12 \mathrm{vg}$ respectively); however, only $30 \mu \mathrm{L}$ and $150 \mu \mathrm{L}$ of vector were delivered to these respective regions. Fifty-one patients were enrolled in this 15- to 24month, multicenter, double-blind trial, but there was no significant difference between groups in the primary endpoint or in most secondary endpoints [53]. These results strongly 
suggest that insufficient or inaccurate delivery of the vector occurred, given the low infusion volumes and lack of realtime MRI.

\section{Huntington's Disease}

Huntington's disease is an autosomal dominant disorder caused by an excessive number of trinucleotide $\mathrm{CAG}$ repeats on exon 1 of chromosome 4, resulting in a polyglutaminated huntingtin ( $\mathrm{mHtt}$ ) protein [55]. Ultimately, increased levels of $\mathrm{mHtt}$ aggregate within striatal medium spiny neurons and pyramidal cortical neurons and are responsible for the pathological phenotype observed in HD. Patients often present around the age of 40 with motor, cognitive, and psychiatric symptoms. Motor symptoms vary by the progression of the disease. Early on, patients display choreiform movements with gait impairment which progress to bradykinesia and rigidity [56]. Cognitive changes include deficits in attention and mental flexibility, whereas psychiatric manifestations include depression, apathy, and impulsivity [57].

A popular experimental therapeutic approach to address the underlying pathology has been to design drugs that decrease levels of $\mathrm{mHtt}$ in an effort to halt disease progression [58]. Gene therapy is an attractive treatment option for HD, as it allows for direct targeting of dysfunctional nuclei in an effort to reduce levels of $\mathrm{mHtt}$. Preclinical gene therapy strategies for HD have evolved and now are centered around using RNA interference (RNAi) to suppress pathologic levels of $\mathrm{mHtt}$. RNAi involves the use of small RNA molecules such as short interfering RNA, short hairpin RNA, and microRNA to target mRNA molecules and cause them to be destroyed by the RNA-induced silencing complex [59]. These techniques allow for precise targeting of the mHtt mRNA and reduce the likelihood of off-target adverse effects. In addition, nonhuman primate studies have demonstrated that reductions in normal Htt of up to $45 \%$ may be well tolerated [60,61]. An engineered microRNA targeting human huntingtin, delivered via AAV5 with a transgene encoding an engineered miRNA against HTT mRNA (AAV5-miHTT), has been developed [62-64]. Widespread distribution of the transgene has been observed, as would be expected from the properties of AAV5 [5], and as would be desired for this disorder. At the time of writing, this work has not progressed to a clinical stage.

\section{Alzheimer's Disease}

Although Alzheimer's disease (AD) is the most common cause of dementia worldwide, its underlying pathophysiology is less well understood than in PD and HD. AD is associated with loss of cholinergic neurons in the nucleus basalis of Meynert, and cholinesterase inhibitors remain the primary treatment offered to patients with AD but provide only modest symptomatic improvement overall. The function of cholinergic neurons in the basal forebrain is augmented by nerve growth factor (NGF), whose protective effects have been demonstrated in aged nonhuman primates and those with lesions [65-67].

Gene delivery of NGF using AAV2 was initially studied in a dose-escalating phase 1 clinical trial in patients with mild to moderate $\mathrm{AD}$, in which long-term safety and feasibility via a traditional surgical approach was demonstrated. The results of the follow-up, multicenter (10 US academic medical centers) phase 2 trial, was recently reported [14]. That study enrolled 49 participants with mild to moderate $\mathrm{AD}$ who were randomly assigned (1:1) to sham surgery or to receive intracerebral injections of AAV2-NGF. These infusions were undertaken without using CED and without using iMRI guidance. Although this procedure was safe and well tolerated through 24 months, no significant difference was observed between the treatment and placebo groups on the primary outcome measure, the Alzheimer Disease Assessment Scale-cognitive subscale. The study authors noted that pathological confirmation of accurate gene targeting is needed, which is especially true given that infusions occurred in a black box, without any verification that adequate vector was delivered, as could have been realized with iMRI-CED.

\section{Conclusions}

Therapeutic strategies for gene replacement or addition in neurodegenerative brain disorders continue to evolve as advances are made in understanding critical aspects of the underlying molecular pathophysiology. With the advent of iMRI-CED, which allows reliable delivery of therapeutic vectors with real-time verification, the neurosurgical field now stands ready to maximize the potential translation of these strategies for clinical efficacy.

Required Author Forms Disclosure forms provided by the authors are available with the online version of this article.

Publisher's Note Springer Nature remains neutral with regard to jurisdictional claims in published maps and institutional affiliations.

\section{References}

1. Richardson RM, Varenika V, Forsayeth JR, Bankiewicz KS (2009) Future applications: gene therapy. Neurosurg Clin N Am 20:205-210

2. Palfi S, Gurruchaga JM, Ralph GS, et al (2014) Long-term safety and tolerability of ProSavin, a lentiviral vector-based gene therapy for Parkinson's disease: a dose escalation, open-label, phase 1/2 trial. Lancet 383:1138-1146 
3. Salegio EA, Samaranch L, Kells AP, et al (2013) Axonal transport of adeno-associated viral vectors is serotype-dependent. Gene Therapy 20:348-352

4. Ciesielska A, Mittermeyer G, Hadaczek P, Kells AP, Forsayeth J, Bankiewicz KS (2011) Anterograde Axonal Transport of AAV2GDNF in Rat Basal Ganglia. Mol Ther 19:922-927

5. Samaranch L, Blits B, San Sebastian W, et al (2017) MR-guided parenchymal delivery of adeno-associated viral vector serotype 5 in non-human primate brain. Gene Ther doi: https://doi.org/10.1038/ gt.2017.14

6. Green F, Samaranch L, Zhang HS, et al (2016) Axonal transport of AAV9 in nonhuman primate brain. Gene Ther 23:520-526

7. San Sebastian W, Samaranch L, Heller G, et al (2013) Adenoassociated virus type 6 is retrogradely transported in the nonhuman primate brain. Gene Ther 20:1178-1183

8. Samaranch L, Salegio EA, San Sebastian W, et al (2013) Strong cortical and spinal cord transduction after AAV7 and AAV9 delivery into the cerebrospinal fluid of nonhuman primates. Hum Gene Ther 24:526-532

9. Kells AP, Hadaczek P, Yin D, et al (2009) Efficient gene therapybased method for the delivery of therapeutics to primate cortex. PNAS 106:2407-2411

10. Eberling JL, Jagust WJ, Christine CW, et al (2008) Results from a phase I safety trial of hAADC gene therapy for Parkinson disease. Neurology 70:1980-1983

11. Marks WJ, Ostrem JL, Verhagen L, et al (2008) Safety and tolerability of intraputaminal delivery of CERE-120 (adeno-associated virus serotype 2-neurturin) to patients with idiopathic Parkinson's disease: an open-label, phase I trial. Lancet Neurol 7:400-408

12. Christine CW, Starr PA, Larson PS, et al (2009) Safety and tolerability of putaminal AADC gene therapy for Parkinson disease. Neurology 73:1662-1669

13. Muramatsu S, Fujimoto K, Kato S, et al (2010) A phase I study of aromatic L-amino acid decarboxylase gene therapy for Parkinson's disease. Mol Ther 18:1731-1735

14. Rafii MS, Tuszynski MH, Thomas RG, et al (2018) AdenoAssociated Viral Vector (Serotype 2)-Nerve Growth Factor for Patients With Alzheimer Disease: A Randomized Clinical Trial. JAMA Neurol 75:834-841

15. Marks WJ, Bartus RT, Siffert J, et al (2010) Gene delivery of AAV2-neurturin for Parkinson's disease: a double-blind, randomised, controlled trial. Lancet Neurol 9:1164-1172

16. LeWitt PA, Rezai AR, Leehey MA, et al (2011) AAV2-GAD gene therapy for advanced Parkinson's disease: A double-blind, shamsurgery controlled, randomised trial. Lancet Neurol 10:309-319

17. Bobo RH, Laske DW, Akbasak A, Morrison PF, Dedrick RL, Oldfield EH (1994) Convection-enhanced delivery of macromolecules in the brain. Proc Natl Acad Sci U S A 91:2076-2080

18. Krauze MT, Saito R, Noble C, et al (2005) Reflux-free cannula for convection-enhanced high-speed delivery of therapeutic agents. J Neurosurg 103:923-929

19. Varenika V, Dickinson P, Bringas J, et al (2008) Detection of infusate leakage in the brain using real-time imaging of convection-enhanced delivery. J Neurosurg 109:874-880

20. Varenika V, Kells AP, Valles F, Hadaczek P, Forsayeth J, Bankiewicz KS (2009) Controlled dissemination of AAV vectors in the primate brain. Prog Brain Res 175:163-172

21. Valles F, Fiandaca MS, Bringas J, et al (2009) Anatomic compression caused by high-volume convection-enhanced delivery to the brain. Neurosurgery 65:579-585; discussion 585-586

22. Valles F, Fiandaca MS, Eberling JL, et al (2010) Qualitative imaging of adeno-associated virus serotype 2-human aromatic L-amino acid decarboxylase gene therapy in a phase $\mathrm{i}$ study for the treatment of parkinson disease. Neurosurgery 67:1377-1385

23. Yin D, Richardson RM, Fiandaca MS, et al (2010) Cannula placement for effective convection-enhanced delivery in the nonhuman primate thalamus and brainstem: implications for clinical delivery of therapeutics. J Neurosurg 113:240-248

24. Yin D, Valles FE, Fiandaca MS, et al (2011) Optimal region of the putamen for image-guided convection-enhanced delivery of therapeutics in human and non-human primates. Neuroimage 54 Suppl 1:S196-203

25. Richardson RM, Kells AP, Martin AJ, et al (2011) Novel platform for MRI-guided convection-enhanced delivery of therapeutics: Preclinical validation in nonhuman primate brain. Stereotact Funct Neurosurg 89:141-151

26. Su X, Kells AP, Salegio EA, et al (2010) Real-time MR imaging with Gadoteridol predicts distribution of transgenes after convection-enhanced delivery of AAV2 vectors. Mol Ther 18: 1490-1495

27. Richardson RM, Kells AP, Rosenbluth KH, et al (2011) Interventional MRI-guided putaminal delivery of AAV2-GDNF for a planned clinical trial in Parkinson's disease. Mol Ther 19: $1048-1057$

28. Larson PS, Starr PA, Bates G, Tansey L, Richardson RM, Martin AJ (2012) An optimized system for interventional magnetic resonance imaging-guided stereotactic surgery: preliminary evaluation of targeting accuracy. Neurosurgery 70:95-103; discussion 103

29. Ostrem JL, Ziman N, Galifianakis NB, et al (2016) Clinical outcomes using ClearPoint interventional MRI for deep brain stimulation lead placement in Parkinson's disease. J Neurosurg 124:908916

30. Sidiropoulos C, Rammo R, Merker B, et al (2016) Intraoperative MRI for deep brain stimulation lead placement in Parkinson's disease: 1 year motor and neuropsychological outcomes. J Neurol 263: $1226-1231$

31. Lee PS, Weiner GM, Corson D, et al (2018) Outcomes of Interventional-MRI Versus Microelectrode Recording-Guided Subthalamic Deep Brain Stimulation. Front Neurol doi: https:// doi.org/10.3389/fneur.2018.00241

32. Levy R, Lang AE, Dostrovsky JO, et al (2001) Lidocaine and muscimol microinjections in subthalamic nucleus reverse Parkinsonian symptoms. Brain 124:2105-2118

33. Emborg ME, Carbon M, Holden JE, et al (2007) Subthalamic glutamic acid decarboxylase gene therapy: changes in motor function and cortical metabolism. J Cereb Blood Flow Metab 27:501509

34. Kaplitt MG, Feigin A, Tang C, et al (2007) Safety and tolerability of gene therapy with an adeno-associated virus (AAV) borne GAD gene for Parkinson's disease: an open label, phase I trial. Lancet 369:2097-2105

35. Bankiewicz KS, Forsayeth J, Eberling JL, et al (2006) Long-Term Clinical Improvement in MPTP-Lesioned Primates after Gene Therapy with AAV-hAADC. Mol Ther 14:564-570

36. Hadaczek P, Eberling JL, Pivirotto P, Bringas J, Forsayeth J, Bankiewicz KS (2010) Eight years of clinical improvement in MPTP-lesioned primates after gene therapy with AAV2-hAADC. Mol Ther 18:1458-61

37. Forsayeth JR, Eberling JL, Sanftner LM, et al (2006) A DoseRanging Study of AAV-hAADC Therapy in Parkinsonian Monkeys. Mol Ther 14:571-577

38. San Sebastian W, Richardson RM, Kells AP, et al (2012) Safety and tolerability of magnetic resonance imaging-guided convection-enhanced delivery of AAV2-hAADC with a novel delivery platform in nonhuman primate striatum. Hum Gene Ther 23:210-217

39. Bankiewicz KS, Eberling JL, Kohutnicka M, et al (2000) Convection-enhanced delivery of AAV vector in parkinsonian monkeys; in vivo detection of gene expression and restoration of dopaminergic function using pro-drug approach. Exp Neurol 164: $2-14$ 
40. Mittermeyer G, Christine CW, Rosenbluth KH, et al (2012) Longterm evaluation of a phase 1 study of AADC gene therapy for Parkinson's disease. Hum Gene Ther 23:377-381

41. Dass B, Olanow CW, Kordower JH (2006) Gene transfer of trophic factors and stem cell grafting as treatments for Parkinson's disease. Neurology 66:S89-S103

42. Lang AE, Gill S, Patel NK, et al (2006) Randomized controlled trial of intraputamenal glial cell line-derived neurotrophic factor infusion in Parkinson disease. Ann Neurol 59:459-466

43. Gill SS, Patel NK, Hotton GR, et al (2003) Direct brain infusion of glial cell line-derived neurotrophic factor in Parkinson disease. Nat Med 9:589-595

44. Slevin JT, Gerhardt GA, Smith CD, Gash DM, Kryscio R, Young B (2005) Improvement of bilateral motor functions in patients with Parkinson disease through the unilateral intraputaminal infusion of glial cell line - derived neurotrophic factor. J Neurosurg 102:216222

45. Salvatore MF, Ai Y, Fischer B, et al (2006) Point source concentration of GDNF may explain failure of phase II clinical trial. Exp Neurol 202:497-505

46. Barua N, Gill S (2018) Drug Delivery for Movement Disorders. Curr Concepts Mov Disord Manag 33:243-252

47. Kordower JH, Emborg ME, Bloch J, et al (2000) Neurodegeneration prevented by lentiviral vector delivery of GDNF in primate models of Parkinson's disease. Science 290: $767-73$

48. Johnston LC, Eberling J, Pivirotto P, et al (2009) Clinically Relevant Effects of Convection-Enhanced Delivery of AAV2GDNF on the Dopaminergic Nigrostriatal Pathway in Aged Rhesus Monkeys. Hum Gene Ther 20:497-510

49. Su X, Kells AP, Huang EJ, et al (2009) Safety evaluation of AAV2GDNF gene transfer into the dopaminergic nigrostriatal pathway in aged and parkinsonian rhesus monkeys. Hum Gene Ther 20:16271640

50. Kells AP, Eberling J, Su X, et al (2010) Regeneration of the MPTPLesioned Dopaminergic System after Convection-Enhanced Delivery of AAV2-GDNF. J Neurosci 30:9567-9577

51. Cik M, Masure S, Lesage ASJ, et al (2000) Binding of GDNF and Neurturin to Human GDNF family receptor alpha 1 and 2: Influence of cRET and cooperative interactions. J Biol Chem 275: 27505-27512

52. Kordower JH, Herzog CD, Dass B, et al (2006) Delivery of neurturin by AAV2 (CERE-120)-mediated gene transfer provides structural and functional neuroprotection and neurorestoration in MPTP-treated monkeys. Ann Neurol 60:706-715

53. Olanow WC, Bartus RT, Baumann TL, et al (2015) Gene delivery of neurturin to putamen and substantia nigra in Parkinson disease:
A double-blind, randomized, controlled trial. Ann Neurol 78:248257

54. Bartus RT, Baumann TL, Siffert J, et al (2013) Safety/feasibility of targeting the substantia nigra with AAV2-neurturin in Parkinson patients. Neurology 80:1698-1701

55. MacDonald ME, Ambrose CM, Duyao MP, et al (1993) A novel gene containing a trinucleotide repeat that is expanded and unstable on Huntington's disease chromosomes. Cell 72:971-983

56. Ross CA, Tabrizi SJ (2011) Huntington's disease: From molecular pathogenesis to clinical treatment. Lancet Neurol 10:83-98

57. Saudou F, Humbert S (2016) The Biology of Huntingtin. Neuron 89:910-926

58. Yamamoto A, Lucas JJ, Hen R (2000) Reversal of Neuropathology and Motor Dysfunction in a Conditional Model of Huntington's Disease. Cell 101:57-66

59. Hutvagner G, Simard MJ (2008) Argonaute proteins: Key players in RNA silencing. Nat Rev Mol Cell Biol 9:22-32

60. McBride JL, Pitzer MR, Boudreau RL, et al (2011) Preclinical safety of RNAi-mediated HTT suppression in the rhesus macaque as a potential therapy for Huntington's disease. Mol Ther 19:21522162

61. Grondin R, Kaytor MD, Ai Y, et al (2012) Six-month partial suppression of Huntingtin is well tolerated in the adult rhesus striatum. Brain 135:1197-1209

62. Miniarikova J, Zanella I, Huseinovic A, et al (2016) Design, Characterization, and Lead Selection of Therapeutic miRNAs Targeting Huntingtin for Development of Gene Therapy for Huntington's Disease. Mol Ther Nucleic Acids 5:e297

63. Miniarikova J, Zimmer V, Martier R, et al (2017) AAV5-miHTT gene therapy demonstrates suppression of mutant huntingtin aggregation and neuronal dysfunction in a rat model of Huntington's disease. Gene Ther 24:630-639

64. Evers MM, Miniarikova J, Juhas S, et al (2018) AAV5-miHTT Gene Therapy Demonstrates Broad Distribution and Strong Human Mutant Huntingtin Lowering in a Huntington's Disease Minipig Model. Mol Ther 26:2163-2177

65. Koliatsos VE, Nauta HJ, Clatterbuck RE, Holtzman DM, Mobley WC, Price DL (1990) Mouse nerve growth factor prevents degeneration of axotomized basal forebrain cholinergic neurons in the monkey. J Neurosci 10:3801-3813

66. Kordower JH, Winn SR, Liu YT, et al (1994) The aged monkey basal forebrain: rescue and sprouting of axotomized basal forebrain neurons after grafts of encapsulated cells secreting human nerve growth factor. Proc Natl Acad Sci U S A 91:10898-10902

67. Tuszynski MH, Roberts J, Senut MC, U HS, Gage FH (1996) Gene therapy in the adult primate brain: intraparenchymal grafts of cells genetically modified to produce nerve growth factor prevent cholinergic neuronal degeneration. Gene Ther 3:305-314 